\title{
LESÕES CERVICAIS NÃO CARIOSAS: UM PANORAMA ATUAL
}

\section{NON-CARIOUS CERVICAL LESIONS: A CURRENT VIEW}

\author{
Alidianne Fábia Cabral Xavier* \\ Tássia Cristina de Almeida Pinto** \\ Alessandro Leite Cavalcanti"*
}

\begin{abstract}
RESUMO
A perda progressiva da estrutura dentária associada à ocorrência de lesões cervicais não cariosas tem-se mostrado um problema cada vez mais frequente na prática odontológica contemporânea, uma vez que o aumento da expectativa de vida tem favorecido a manutenção da dentição natural. Dessa forma, o número de pessoas que expõem seus dentes por um maior período de tempo aos múltiplos fatores etiológicos relacionados à perda progressiva e não cariosa das estruturas dentais também tem aumentado, fato que pode resultar em complicações estéticas e funcionais. Portanto, este trabalho destina-se a realizar uma revisão de literatura acerca dos fatores etiológicos, classificação, características clínicas e opções terapêuticas para as lesões cervicais não cariosas, possibilitando aos cirurgiões-dentistas o estabelecimento do diagnóstico diferencial, de condutas preventivas e de tratamento.
\end{abstract}

DESCRITORES: Erosão dentária • Abrasão dentária • Desgaste dos dentes • Dentística operatória

\section{ABSTRACT}

The progressive loss of tooth structure associated with the occurrence of non-carious cervical lesions has been an increasingly frequent problem in contemporary dental practice, since the increase in life expectancy has favored the maintenance of the natural dentition. Thus, the number of people who expose their teeth to a longer period of time to multiple etiologic factors related to the gradual loss of non-carious dental structures has also increased, which may result in aesthetic and functional complications. Therefore, this work is intended to perform a literature review about the etiological factors, classification, clinical features and therapeutic options of non-carious cervical lesions, in order to enable such information of differential diagnosis, preventive measures and treatment to dentists.

DESCRIPTORS: Tooth erosion • Tooth abrasion • Tooth wear • Operative dentistry

\footnotetext{
* Mestranda em Clínica Odontológica da Universidade Estadual da Paraíba (UEPB), Campina Grande/PB, Brasil, alidianne.fabia@gmail.com.

** Mestranda em Clínica Odontológica da Universidade Estadual da Paraíba (UEPB), Campina Grande/PB, Brasil, tassiapinto@yahoo.com.br.

*** Professor Doutor do Programa de Pós-Graduação em Odontologia da Universidade Estadual da Paraíba (UEPB), Campina Grande/PB, Brasil, dralessandro@ ibest.com.br.
} 
XAVIER AFC

PINTO TCA CAVALCANTI AL LESÕES CERVICAIS NÃO CARIOSAS: UM PANORAMA ATUAL

REV, ODONTOL, UNIV. CID. SÃO PAULO $2012 ; 24(1): 57-$ 66 , JAN-ABR

\section{N T R O D UÇ Ã O}

As patologias cervicais são divididas em lesões cariosas e não cariosas, cuja ocorrência gera perda irreversível da estrutura dentária. Entretanto, enquanto as lesões cariosas são originadas por processos que envolvem microrganismos, as lesões não cariosas possuem causa etiológica multifatorial e não bacteriana Kliemann ${ }^{1}$, (2002), sendo classificadas em abfração, erosão e abrasão (Grippo et al. ${ }^{2}$, 2004).

A prevalência de cárie e de lesões não cariosas foi investigada em populações arqueológicas na América do Norte e na Europa, verificando-se que houve um predomínio de lesões não cariosas nas populações mexicanas, não sendo encontrada nenhuma associação estatisticamente significativa entre esse tipo de lesão e a idade, gênero e dieta (Ritter et al. ${ }^{3}, 2009$ ).

Os problemas associados com a perda progressiva e não cariosa da estrutura dental estão se tornando um problema crescente para a Odontologia, uma vez que as pessoas estão mantendo sua dentição natural até idades mais avançadas e com isso expõem seus dentes aos fatores etiológicos por um período de tempo maior que os pacientes jovens (Marochi e Queiroz ${ }^{4}$, 2001).

Conhecer o quanto cada agente etiológico está contribuindo em uma determinada etapa no processo da lesão instalada é fundamental para tratar e prevenir futuras lesões (Barbosa et al. ${ }^{5}, 2009$ )

Por meio de uma investigação acerca da prevalência de lesões cervicais não cariosas (LCNC) em idosos e sua correlação com hábitos de higiene, parafuncionais e de dieta, Molena et al. ${ }^{6}$ (2008) observaram em $77 \%$ dos pacientes pelo menos uma LCNC, mas sem qualquer correlação com os fatores etiológicos de cada lesão específica.

Inúmeros possíveis fatores etiológicos como ácidos, tensões oclusais e processos abrasivos têm sido sugeridos para explicar o surgimento das LCNC, sendo constatado que, quando esses processos estão presentes simultaneamente, a incidência de lesões é elevada (Lee e Eakle7, 1984).

De modo geral, os dentes superiores mais comumente afetados são os primeiros pré-molares, seguidos pelos primeiros molares, segundos pré-molares e caninos. Quanto aos dentes inferiores, os primeiros pré-molares novamente são os mais frequentemente acometidos, seguidos pelos segundos pré-molares, primeiros molares e caninos (Telles et al. ${ }^{8}, 2000$ ).

No estudo realizado na Universidade Federal de Sergipe com pacientes de idade entre 18 e 64 anos, observou-se que a distribuição das lesões de acordo com o dente acometido ocorreu da seguinte forma: $62,16 \%$ nos pré-molares, $24,32 \%$ nos molares e, em menor proporção, 6,76\% nos incisivos e caninos (Oliveira et al. ${ }^{9}$, 2010).

Essas lesões são mais comumente diagnosticadas pela anamnese detalhada e pelo exame visual e tátil. No entanto, torna-se oportuno ressaltar que falhas na detecção dos possíveis fatores causais podem resultar em aumento da retenção de placa bacteriana, perda continuada e enfraquecimento da estrutura dental, comprometimento da estética e sensibilidade dental, necessidade de tratamento endodôntico ou perda do dente e a ocorrência de novas lesões em outros dentes (Michael et al. ${ }^{10}, 2010$ ).

Desse modo, destaca-se a importância do reconhecimento das lesões ainda em estágio inicial, para que assim sejam possíveis a identificação dos fatores etiológicos e a realização de um diagnóstico diferencial, permitindo que haja a implementação de um programa preventivo para evitar a evolução do quadro ou o aparecimento de novas lesões e, sobretudo, definir um plano de tratamento para cada caso em particular (Pegoraro et al. ${ }^{11}$, 2000).

Diante do exposto, este estudo tem como objetivo realizar uma revisão de literatura sobre fatores etiológicos, classificação, características clínicas e opções terapêuticas das lesões cervicais não cariosas.

\section{REVISÃO DE LITERATURA}

\section{Abfração}

A palavra abfração deriva do verbo latino frango, fregi, fractum (quebrar) e é uti- 
lizado para descrever uma perda patológica dos tecidos duros dentários, oriunda de forças oclusais traumáticas que provocam flexões dentais, promovendo alterações do esmalte, dentina e cemento, distantes do local do trauma de oclusão (Paiva et al. $\left.{ }^{12}, 2003\right)$.

A etiologia da abfração é considerada multifatorial, uma vez que esse esforço excessivo pode originar-se de interferências oclusais, do apertamento ou até mesmo de esforço mastigatório, principalmente quando forças oclusais excêntricas são introduzidas (Smith et al. ${ }^{13}$, 2008).

De acordo com a teoria da flexão do dente, forças parafuncionais em áreas em que ocorrem interferências podem proporcionar a um ou mais dentes fortes esforços tensionais, compressivos ou de cisalhamento. Essas forças se concentram na junção cemento-esmalte, promovendo microfraturas nesse tecido, através das quais moléculas de saliva e água penetram, tornando a região suscetível ao efeito solubilizador de ácidos e efeito abrasivo da escovação. Acredita-se que, com o tempo, as microfraturas evoluem perpendicularmente ao longo eixo dos dentes sob pressão até o esmalte e a dentina serem "fraturados", culminando com defeitos em forma de cunha com bordas afiadas (Pereira' $\left.{ }^{14}, 1995\right)$.

Esse tipo de lesão tem a possibilidade de ser o fator inicial e progressivo dominante na produção das LCNC. As forças oclusais repetitivas e estáticas quando em combinação com um agente corrosivo, abrasivo, ou ambos, podem promover um efeito altamente significativo (Hanaoka et al. $\left.{ }^{15}, 1998\right)$.

Clinicamente, as abfrações apresentam-se em forma de cunha, geralmente profundas e com margem definida Schneider ${ }^{16}$, (s.d.), exibem tamanho proporcional à magnitude e frequência de aplicação da força de tração nos elementos dentários (Lee e Eakle ${ }^{7}$, 1984). A maior incidência tem sido observada nos dentes inferiores, possivelmente devido ao menor diâmetro coronário na região cervical desses dentes Schneider ${ }^{16}$, (s.d.). Segundo Rees et al. ${ }^{17}$ (2003), os dentes mais acometidos pela abfração são os pré-molares superiores e inferiores, seguidos dos caninos e, em me- nor proporção, dos incisivos superiores, que raramente são relatados na literatura.

A abfração acomete dentes periodontalmente sadios, geralmente afetando um único dente, pois se acredita que a presença de mobilidade dental impede a instalação desse tipo de lesão (Paiva et al. ${ }^{12}$, 2003). Isso ocorre porque dentes periodontalmente comprometidos, quando sob a ação de forças oclusais mal direcionadas, movimentam-se em seus alvéolos praticamente sem resistência e, dessa forma, não há concentração de tensões (Garone-Filho e Silva ${ }^{18}$ 2008).

Por outro lado, em pacientes mais idosos, o periodonto se torna mais rígido, o que aumenta a incidência de lesões cervicais anguladas, pois a capacidade de um periodonto de absorver parte dos esforços oclusais é perdida (Garone-Filho e Silva ${ }^{18}$ 2008).

A intervenção restauradora no dente com lesão de abfração deve ser precedida da correção oclusal através da eliminação das interferências oclusais, tanto nos movimentos de testes, lateralidade e protrusão, como nos movimentos funcionais, principalmente os ciclos mastigatórios (Bataglion e Nunes ${ }^{19}$, 1999).

Dependendo da quantidade de estrutura dental perdida, presença de sensibilidade e do envolvimento estético, pode-se optar pela aplicação de agentes dessensibilizantes, laserterapia, restaurações em cimento de ionômero de vidro, resina composta ou amálgama de prata ou, mesmo, a associação deles (Hoeppner et al. ${ }^{20}$, 2007).

\section{Erosão}

A erosão dental é definida como a perda de substância dentária por processos químicos (exposição ácida) sem o envolvimento de bactérias. O ataque ácido conduz a uma perda irreversível do tecido duro dental, que é acompanhado por um amolecimento progressivo da superfície (Lussi ${ }^{21}$, 2006).

O processo se inicia com a desmineralização das camadas superficiais do esmalte, podendo evoluir para uma perda considerável de estrutura dental, visto que qualquer substância ácida com $\mathrm{pH}$ inferior ao crítico para o esmalte $(5,5)$ e
XAVIER AFC

PINTO TCA

CAVALCANTI AL

LESÕES

CERVICAIS NÃO

CARIOSAS: UM

PANORAMA ATUAL
REV, ODONTOL.

UNIV. CID, SÃo PAULO

2012; $24(1): 57$. 66 , JAN-ABR 
XAVIER AFC

PINTO TCA

CAVALCANTI AL LESÕES CERVIC-

AIS NÃO CARI-

OSAS: UM PANO-

RAMA ATUAL

\section{0}

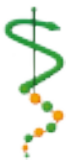

REV, ODONTOL.

UNIV. CID . SÃO

PAULO

$2012 ; 24(1): 57-$

66, JAN-ABR dentina $(4,5)$ pode dissolver os cristais de hidroxiapatita (Grippo et al. ${ }^{2}, 2004$, Barron et al.22, 2003).

As lesões erosivas caracterizam-se pela descalcificação pouco profunda do esmalte, manchas brancas, dureza e aspereza superficiais, cavidades largas, rasas e sem ângulos nítidos (Sobral et al. ${ }^{23}, 2000$ ). O esmalte erosionado mostra-se sem briIho, fosco, com degraus e concavidades. As cúspides encontram-se arredondadas e algumas concavidades alcançam a dentina, bem como, em dentes restaurados, os limites do material restaurador estão acima da superfície dentária subjacente (Lussi et al. $\left.{ }^{24}, 1995\right)$.

Esse tipo de desgaste dental pode ser desencadeado por fatores intrínsecos, como a xerostomia, anorexia nervosa, bulimia e problemas gastroesofágicos cujas frequentes regurgitações e vômitos expõem o ambiente bucal a um pH de aproximadamente 2,3 pela presença do suco gástrico. Os fatores extrínsecos, que também podem ser responsáveis pela ocorrência da erosão, incluem alimentos, bebidas (refrigerantes, sucos de frutas, isotônicos e chás) e produtos ácidos advindos do ambiente de trabalho, os quais mantêm o pH bucal baixo (Lussi et al. ${ }^{25}, 1993$, Lussi et al. ${ }^{26}, 2004$, Xavier et al. $\left.{ }^{27}, 2010\right)$.

Dessa forma, o desenvolvimento do processo erosivo apresenta etiologia multifatorial, modulada por fatores determinantes químicos, biológicos e comportamentais Lussi' ${ }^{21}$, (2006), Lussi et al. ${ }^{28}$, (2007), por modificadores, como o conhecimento e condições socioeconômicas Lussi et al. ${ }^{28},(2007)$, sendo influenciada por hábitos pessoais (Silva et al. ${ }^{29}, 2007$ ). A erosão é considerada a forma mais comum de desgaste na infância May e Waterhouse ${ }^{30}$, (2003) e poderá se tornar um dos maiores problemas de saúde pública em pessoas jovens (Truin et al. ${ }^{31}, 2005$ ).

Quanto ao tratamento, a primeira medida terapêutica a ser adotada consiste na remoção dos fatores causais e, posteriormente, na análise individual de aspectos como a profundidade da lesão e a presença de sintomatologia dolorosa para determinar a necessidade ou não de procedimento restaurador $\left(\right.$ Braga $\left.^{32}, 2005\right)$. Caso esse seja necessário, diversos materiais, como as resinas compostas, cimentos de ionômero de vidro convencionais, cimentos de ionômero de vidro modificados por resina e compômeros podem ser usados para o restabelecimento da estrutura dentária, da função e da estética, bem como para controlar a hipersensibilidade dentinária (Jaeggi et al.33, 2006).

A longevidade das restaurações depende da durabilidade do material e de suas propriedades, tais como a resistência ao desgaste, degradação de interface entre dente e restauração e do nível de destruição dentária. Sob condições ácidas, todos os materiais restauradores dentários têm mostrado degradação ao longo do tempo (Jaeggi et al. $\left.{ }^{33}, 2006\right)$.

\section{Abrasão}

As lesões cervicais não cariosas, provocadas por abrasão, são definidas como o desgaste patológico dos tecidos duros dentais por forças mecânicas não relacionadas com a oclusão $\left(\right.$ Braga $^{32}$, 2005). Essa perda da substância dental ocorre através de um processo mecânico repetitivo que envolve objetos ou substâncias, podendo ser difusa ou localizada (Esteves ${ }^{34}, 2010$ ).

A abrasão acontece quando uma superfície áspera e dura desliza ao longo de uma superfície com um menor grau de dureza, cortando-a ou sulcando-a na forma de uma série de ranhuras (Kliemann ${ }^{1}$ 2002). Os fatores envolvidos na sua ocorrência incluem objetos estranhos ou substâncias constantemente introduzidas na boca e que mantêm contato com o dente (Imfeld $\left.{ }^{35}, 1996\right)$.

Os principais fatores etiológicos relacionados são procedimentos de higienização bucal, além daqueles relativos ao paciente, os quais envolvem técnica, frequência, tempo e força aplicada durante o processo de escovação. Em superfícies proximais, deve ser considerado ainda o uso de palitos de dente e fio dental, principalmente quando estes estão associados a uma substância abrasiva (Imfeld $\left.{ }^{35}, 1996\right)$. Além disso, as lesões de abrasão podem estar associadas à raspagem e curetagem periodontal (Schneider ${ }^{16}$, s.d.).

Clinicamente, a lesão é caracterizada por apresentar uma superfície dura, altamente polida, rasa, com contornos 
regulares, verificando-se, nos dentes proeminentes, caninos e pré-molares, um maior grau de perda de estrutura dentária (Schneider ${ }^{16}$, s.d.).

Assim, com maior frequência e como medida preventiva ao aparecimento da lesão, a conduta básica é a orientação quanto ao uso correto da escova dental e a escolha de um dentifrício pouco abrasivo (Hoeppner et al. $\left.{ }^{20}, 2007\right)$.

A presença de sensibilidade, decorrente da exposição do tecido dentinário, pode ser tratada com a aplicação de agentes dessensibilizantes, com laserterapia ou mesmo com a confecção de restaurações em cimento de ionômero de vidro, resina composta ou amálgama de prata, desde que a quantidade de tecido abrasionado permita a inserção desses materiais sem, contudo, alterar o contorno do elemento dental (Hoeppner et al.. $\left.{ }^{20}, 2007\right)$.

\section{I SCUSSÃO}

As medidas preventivas como o emprego abrangente de flúor, tanto no abastecimento de água quanto nos dentifrícios, vêm diminuindo a prevalência da doença cárie. Além disso, o desenvolvimento de materiais e técnicas restauradoras mais adequadas e o maior acesso da população ao consultório odontológico têm propiciado a manutenção dos dentes na cavidade bucal por períodos mais prolongados (Sorensen e Nguyen ${ }^{36}$, 2002). Por outro lado, a Odontologia atual tem-se deparado com outros problemas na cavidade bucal, dentre eles as lesões não cariosas que acometem principalmente a região cervical (Furlan et al. $\left.{ }^{37}, 2005\right)$.

Dessa forma, supõe-se que o número de pessoas que expõem seus dentes por um maior período de tempo a fatores etiológicos relacionados à perda progressiva e não cariosa das estruturas dentais também tem aumentado (Telles ${ }^{38}, 2000$ ).

Assim, para o correto e precoce diagnóstico, o Cirurgião-Dentista deve ater-se ao fato de que os desgastes dentários comumente apresentam etiologia multifatorial, sendo necessária uma minuciosa anamnese e exame clínico apurado para iniciar a terapêutica. Durante esses procedimentos, o profissional deverá identificar possíveis fatores de interferência oclusal e presença de hábitos parafuncionais para, então, traçar um plano de tratamento que poderá englobar orientação dietética, controle psicoemocional, em conjunto com outros profissionais para reabilitar a saúde do paciente Soares et al. ${ }^{39}$, (2006), além de etapas restauradora, oclusal e endodôntica (Dotto et al. ${ }^{40}, 2008$ ).

As características oclusais, a história relatada pelo paciente e as características morfológicas das lesões geralmente norteiam o profissional em direção a um fator etiológico específico (Barron et al..22, 2003). Entretanto, muitos autores em seus trabalhos concluíram que na maioria dos casos existe uma complexa interação dos diferentes fatores que dificultam a identificação de somente uma causa (Hoeppner et al. ${ }^{20}, 2007$, Nguyen et al. ${ }^{41}, 2008$, Tsiggos et al. $\left.{ }^{42}, 2008\right)$.

Devido à complexidade das diversas atividades que ocorrem no meio oral e dos seus efeitos, torna-se difícil classificar cada lesão em uma única categoria, pois inúmeros fatores estão envolvidos no seu desenvolvimento (Grippo e Simring ${ }^{43}$, 1995).

Uma nova classificação das lesões cervicais não cariosas foi proposta por Michael et al. ${ }^{10}$ (2010), os quais estabeleceram como parâmetros de classificação o aspecto morfológico macroscópico apresentado pela lesão. Após a identificação das lesões, foi feita a categorização em cinco grupos, sendo estes: raso, côncavo, em forma de cunha, entalhado e irregular. É importante investigar as mudanças morfológicas dessas lesões no decorrer do tempo, associando a nova classificação das LCNC com a sua etiologia (Michael et al. $\left.{ }^{10}, 2010\right)$.

Dessa forma, se faz necessário ressaltar que, além dos fatores etiológicos extrínsecos, a forma, a posição dos dentes no arco e o $\mathrm{pH}$ da saliva podem influenciar o desgaste do dente. O desenvolvimento e atividade dos músculos da mastigação, juntamente com o estado emocional do indivíduo, podem também ser fatores contribuintes na perda de estrutura dentária (Grippo e Simring ${ }^{43}$, 1995).

O resultado da perda de substância dentária por abrasão durante a escovação
XAVIER AFC

PINTO TCA

CAVALCANTI AL

LESÕES

CERVICAIS NÃO

CARIOSAS: UM

PANORAMA ATUAL

61

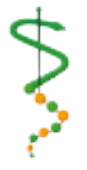

REV, ODONTOL.

UNIV. CID, SÃo

PAULO

2012; $24(1)$ : 57 -

66 , JAN-ABR 
XAVIER AFC

PINTO TCA

CAVALCANTI AL LESÕES CERVIC-

AIS NÃO CARI-

OSAS: UM PANO-

RAMA ATUAL

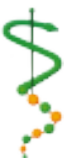

REV, ODONTOL.

UNIV. CID, SÃO

PAULO

$2012 ; 24(1): 57-$

66, JAN-ABR somada à superfície dental erodida é chamada de abrasão-erosão (tensão concentrada na presença de substâncias erosivas). Esse processo ocorre quando a escovação dentária é realizada imediatamente após o consumo de uma bebida ácida, visto que o dente desmineralizado fica mais susceptível à lesão de abrasão por forças mecânicas, como escovação dental (Grippo et al. ${ }^{2}, 2004$, Hooper et al. ${ }^{44}, 2003$, Turssi et al. $\left.{ }^{45}, 2004\right)$. Por sua vez, o termo abrasão-abfração pode ser referido à perda patológica de estrutura dental causada por efeito friccional das escovas dentais sobre a lesão de abfração (Grippo et al.2 ${ }^{2}$ 2004).

As pesquisas apontam valores discrepantes (0 a $85 \%$ ) no que se refere à prevalência de LCNC, sendo essa gama de estimativas atribuída a diferentes desenhos de estudo, às metodologias utilizadas para determinar presença da lesão e ao tamanho das amostras nas populações estudadas (Aubry et al. ${ }^{46}, 2003$ ).

Em estudo acerca das características clínicas de 125 pacientes portadores de LCNC assistidos na disciplina de Dentística Clínica da Universidade Federal da Paraíba, foi constatada uma prevalência de $17 \%$. Verificou-se ainda que $70,6 \%$ destas eram do tipo abrasão e $29,4 \%$ do tipo abfração Santos et al. ${ }^{47}$, (2005), o que difere dos resultados encontrados por Molena et al. ${ }^{6}$ (2008), os quais revelaram uma prevalência de $63 \%$ e $42 \%$, respectivamente.

Por outro lado, em um levantamento feito na Universidade Federal de Sergipe com uma amostra de 100 pacientes com idade entre 18 e 64 anos, observou-se que houve um maior predomínio dessas lesões (44\%), sendo verificada uma média de 3,36 lesões por paciente, estando 98,7\% delas localizadas na face vestibular (Oliveira et al. ${ }^{9}, 2010$ ).

Presume-se que a prevalência é diretamente proporcional à idade dos pacientes, uma vez que a evolução de um processo degradativo dessa natureza sofre influência do tempo em que os estímulos atuam sobre os dentes. Entretanto, um número expressivo de lesões tem sido reportado entre pacientes jovens, com menos de 35 anos $\left(\right.$ Costa $^{48}$, 2007). Há relatos na literatura Negoro et al. ${ }^{49},(1998)$, de que a perda de estrutura dentária é maior em pa- cientes com menos de 26 anos de idade, do que naqueles com faixa etária entre 30 e 55 anos. Nesse mesmo contexto, existem evidências científicas de que não há uma associação estatisticamente significativa entre a ocorrência de LCNC e a idade do paciente (Ritter et al. ${ }^{3}$, 2009).

Em relação ao grupo de dentes, a maior parte da literatura Oliveira et al. ${ }^{9},(2010)$, Barron et al..22, (2003), Santos et al. ${ }^{47}$, (2005), Wood et al. ${ }^{50},(2009)$ relata que os pré-molares são os mais acometidos por lesões não cariosas, seguidos pelos molares e, em menor frequência, os incisivos e os caninos. Entretanto, Telles et al. ${ }^{51}$ (2006) encontraram resultados de maior prevalência entre o grupo dos dentes molares.

Como consequência da abfração, erosão ou abrasão pode haver exposição dentinária, podendo desencadear a hipersensibilidade (Pereira ${ }^{14}$, 1995, Ferreira et al. ${ }^{52}$, 2001). Dessa maneira, os vernizes têm valor clínico no tratamento de LCNC que desencadeiam hipersensibilidade dentinária, principalmente devido à sua ação seladora nos túbulos dentinários, mas seu tempo de efeito é muito curto, pois são rapidamente removidos pela saliva (Vale e Bramante ${ }^{53}$, 1997). Desse modo, atualmente, LCNC são restauradas mais frequentemente com sistemas adesivos, resinas compostas, cimentos de ionômero de vidro e compômeros (Santos et al. $\left.{ }^{47}, 2005\right)$.

Torna-se oportuno ressaltar que, em lesões de pequena magnitude, mesmo quando há hipersensibilidade associada, o profissional deve tomar medidas preferencialmente não restauradoras, como o uso de vernizes que possibilitam a obliteração dos canalículos dentinários ou realização de ajuste oclusal (Conceição ${ }^{54}$, 2000).

Reveste-se, portanto, de considerável importância a discussão e compreensão da etiologia e da prevalência das LCNC, posto que a dinâmica que envolve o surgimento e estabelecimento dessas lesões influencia diretamente a conduta terapêutica a ser empregada pelo profissional, sobretudo devido ao fato de que qualquer recurso utilizado para tratamento somente terá seu efeito permanente se houver um diagnóstico preciso e controle dos fatores 
responsáveis pela degradação das estruturas mineralizadas do dente.

\section{CONSI DERAÇÕES FINA IS}

Diante do exposto, percebe-se que as lesões cervicais não cariosas apresentam grande variedade de forma e fatores etiológicos diversos que podem atuar de for- ma isolada ou em associação, com intensidade, duração e frequência variáveis, o que as caracteriza como uma condição de natureza multifatorial, que pode se manifestar em todos os grupos etários. Sendo assim, faz-se necessário o estudo de tais eventos para que seja possível prevenir o surgimento de novas lesões e interromper a progressão daquelas já existentes.

\section{REFERÊNCIAS}

1. Kliemann C. Lesões cervicais não-cariosas por abrasão (escovação traumática). J Bras Clin Odontol Int 2002 maio-jun.;6(33):204-9.

2. Grippo JO, Simring M, Schreiner S. Attrition, abrasion, corrosion and abfraction revisited: a new perspective on tooth surface lesions. J Am Dent Assoc 2004 Aug;135(8):1109-18; quiz 63-5.

3. Ritter AV, Grippo JO, Coleman TA, Morgan ME. Prevalence of carious and non-carious cervical lesions in archaeological populations from North America and Europe. J Esthet Restor Dent 2009 21(5):324-34.

4. Marochi C, Queiroz V. Lesões não-cariosas. In: Gomes J, editor. Estética em clínica odontológica. Curitiba: Maio; 2001. p. 151-94.

5. Barbosa L, Prado-Junior R, Mendes R. Lesões cervicais não-cariosas: etiologia e opções de tratamento restaurador. Revista Dentística on line 2009 8(18):5-10.

6. Molena C, Rapoport A, Rezende C, Queiroz C, Denardin O. Lesões não cariosas no idoso. Rev Bras Cir Cabeça Pescoço 2008 jul./set.;37(3):152-5.

7. Lee WC, Eakle WS. Possible role of tensile stress in the etiology of cervical erosive lesions of teeth. J Prosthet Dent 1984 Sep;52(3):374-80.

8. Telles D, Pegoraro LF, Pereira JC. Prevalence of noncarious cervical lesions and their relation to occlusal aspects: a clinical study. J Esthet Dent 2000 12(1):10-5.

9. Oliveira A, Damascena N, Souza C. Análise clínica de pacientes portadores de lesões cervicais não cariosas e sua relação com hábitos. RSBO 2010 jun.;7(2):182-92.

10. Michael JA, Kaidonis JA, Townsend GC. Non-carious cervical lesions on permanent anterior teeth: a new morphological classification. Aust Dent J 2010 Jun;55(2):1347 .

11. Pegoraro C, Sakamoto F, Domingues L. Perimólise: etiologia, diagnóstico e prevenção. Rev APCD 2000 mar./abr.;54(2):156-61.

12. Paiva G, Nunes L, Genovese W, Nasr M, Paiva P, Paiva A. Preparo com laser Er: YAG de lesões dentais cervicais causadas por abfração, abrasão e/ou erosão $J B D$ 2003 jan./mar.;2(5):44-9.

13. Smith WA, Marchan S, Rafeek RN. The prevalence and severity of non-carious cervical lesions in a group of patients attending a university hospital in Trinidad. J Oral Rehabil 2008 Feb;35(2):128-34.

14. Pereira J. Hiperestesia dentinária: aspectos clínicos e formas de tratamento. Maxi-Odonto: Dentística 1995 mar./abr.;1(2):1-23.

XAVIER AFC

PINTO TCA

CAVALCANTI al

LESÕES

CERVICAIS NÃO

CARIOSAS: UM

PANORAMA ATUAL 
XAVIER AFC :

PINTO TCA CAVALCANTI AL LESÕES CERVICAIS NÃO CARIOSAS: UM PANORAMA ATUAL

15. Hanaoka K, Nagao D, Mitusi K, Mitsuhashi A, Sugizaki S, Teranaka T. A biomechanical approach to the etiology and treatment of non-carious dental cervical lesions. Bull Kanagawa Dent Coll 1998 26(2'):103-11.

16. Schneider T. Lesões cervicais e hipersensibilidade dentinária. s.d. [cited 2010 dezembro 2010]; Available from: http://www.webodonto.com/html/artigo02.htm.

17. Rees JS, Hammadeh M, Jagger DC. Abfraction lesion formation in maxillary incisors, canines and premolars: a finite element study. Eur J Oral Sci 2003 Apr;111(2):14954 .

18. Garone-Filho W, Silva V. Lesões não cariosas: o novo desafio da odontologia. São Paulo: Santos; 2008.

19. Bataglion C, Nunes L. Ajuste oclusal por desgaste seletivo. São Paulo: PPA; 1999.

20. Hoeppner M, Massarollo S, Bremm L. Considerações clínicas das lesões cervicais não-cariosas. Publicatio UEPG Cienc Biol Saúde 2007 13(3/4):81-6.

21. Lussi A. Dental erosion: from diagnosis to therapy. Community Dent Oral Epidemiol 2006 34(5):398-9.

22. Barron RP, Carmichael RP, Marcon MA, Sandor GK. Dental erosion in gastroesophageal reflux disease. J Can Dent Assoc 2003 Feb;69(2):84-9.

23. Sobral M, Luz M, Gama-Teixeira A, Garone-Netto N. Influência da dieta líquida ácida no desenvolvimento de erosão dental. Pesquisa Odontológica Brasileira 2000 14(406-10.

24. Lussi A, Jaeggi T, Jaeggi-Scharer S. Prediction of the erosive potential of some beverages. 1995 29(5):349-54.

25. Lussi A, Jaggi T, Scharer S. The influence of different factors on in vitro enamel erosion. Caries Res 1993 27(5):387-93.

26. Lussi A, Jaeggi T, Zero D. The role of diet in the aetiology of dental erosion. Caries Res 200438 Suppl 1(34-44.

27. Xavier A, Cavalcanti A, Montenegro R, Melo J. Avaliacao in vitro da microdureza do esmalte dentario apos exposicao a bebidas isotonicas Pesqui Bras Odontopediatria Clin Integr 2010 maio./ago.;10(2):145-50.

28. Lussi A, Schaffner M, Jaeggi T. Dental erosion: diagnosis and prevention in children and adults. Int Dent J 2007 57(6):385-98.

29. Silva J, Baratieri L, Araújo E, Widmer N. Erosão dental: uma doença dos tempos atuais Clín Int J Braz Dent 2007 3(2):150-60.

30. May J, Waterhouse PJ. Dental erosion and soft drinks: a qualitative assessment of knowledge, attitude and behaviour using focus groups of schoolchildren. A preliminary study. Int J Paediatr Dent 2003 Nov;13(6):425-33.

31. Truin GJ, van Rijkom HM, Mulder J, van't Hof MA. Caries trends 1996-2002 among 6- and 12-year-old children and erosive wear prevalence among 12-year-old children in The Hague. Caries Res 2005 Jan-Feb;39(1):2-8.

32. Braga S. Avaliação de bebidas com baixo pH e da ação simulada sobre os materiais restauradores utilizados em lesões cervicais não-cariosas [Dissertação]. São Paulo: Universidade de São Paulo; 2005.

33. Jaeggi T, Gruninger A, Lussi A. Restorative therapy of erosion. Monogr Oral Sci 2006

\footnotetext{
20(200-14.

REV, ODONTOL. 
34. Esteves C. Lesões cervicais não cariosas [Monografia]. Governador Valadares, MG: Universidade Vale do Rio Doce; 2010.

35. Imfeld T. Dental erosion. Definition, classification and links. Eur J Oral Sci 1996 Apr;104(2 ( Pt 2)):151-5.

36. Sorensen JA, Nguyen HK. Evaluation of toothbrush-induced dentin substrate wear using an in vitro ridged-configuration model. Am J Dent 2002 Nov; 15 Spec No(26B$-32 \mathrm{~B}$.

37. Furlan G, Braga S, Steagall-Junior W, Sobral M. Desgaste dental causado por diferentes cerdas de escovas dentais Rev Inst Ciênc Saúde 2005 out./dez.;23(4):305-8.

38. Telles D. Incidência de lesões cervicais não-cariosas em estudantes de odontologia e sua relação com aspectos oclusais [Tese]. Bauru Universidade de São Paulo; 2000.

39. Soares C, Soares P, Soares P, Menezes M, Santos-Filho P. Lesões de abfração: análise dos fatores etiológicos. Clín Int J Braz Dent 2006 jul./set.;2(3):280-6.

40. Dotto S, Carvalho M, Pagliarin C, Ferreira F, Villa M. Lesão cervical näo-cariosa por abfração - terapêutica endodôntica. Revista Dentística on line 2008 8(17):32-7.

41. Nguyen C, Ranjitkar S, Kaidonis JA, Townsend GC. A qualitative assessment of non-carious cervical lesions in extracted human teeth. Aust Dent J 2008 Mar;53(1):4651.

42. Tsiggos N, Tortopidis D, Hatzikyriakos A, Menexes G. Association between self-reported bruxism activity and occurrence of dental attrition, abfraction, and occlusal pits on natural teeth. J Prosthet Dent 2008 Jul;100(1):41-6.

43. Grippo JO, Simring M. Dental 'erosion' revisited. J Am Dent Assoc 1995 May;126(5):619-20, 23-4, 27-30.

44. Hooper S, West NX, Pickles MJ, Joiner A, Newcombe RG, Addy M. Investigation of erosion and abrasion on enamel and dentine: a model in situ using toothpastes of different abrasivity. J Clin Periodontol 2003 Sep;30(9):802-8.

45. Turssi CP, Faraoni JJ, Rodrigues Jr AL, Serra MC. An in situ investigation into the abrasion of eroded dental hard tissues by a whitening dentifrice. Caries Res 2004 Sep-Oct;38(5):473-7.

46. Aubry M, Mafart B, Donat B, Brau JJ. Brief communication: Study of noncarious cervical tooth lesions in samples of prehistoric, historic, and modern populations from the South of France. Am J Phys Anthropol 2003 May;121(1):10-4.

47. Santos R, Barbosa R, Sales G, Costa J. Análise clínica de pacientes portadores de lesões cervicais. Odonto/ Clín-Científ 2005 jan./abr.;4(1):35-42.

48. Costa L. Prevalência das lesões dentárias não cariosas e sua relação com processos erosivos [Tese]. Universidade de São Paulo2007.

49. Negoro T, Briggs J, Plesh O, Nielsen I, McNeill C, Miller AJ. Bruxing patterns in children compared to intercuspal clenching and chewing as assessed with dental models, electromyography, and incisor jaw tracing: preliminary study. ASDC J Dent Child 1998 Nov-Dec;65(6):449-58, 38.

50. Wood ID, Kassir AS, Brunton PA. Effect of lateral excursive movements on the progression of abfraction lesions. Oper Dent 2009 May-Jun;34(3):273-9.

51. Telles D, Pegoraro LF, Pereira JC. Incidence of noncarious cervical lesions and their relation to the presence of wear facets. J Esthet Restor Dent 2006 18(4):178-83; discussion 84.

XAVIER AFC

pinto tCa

CAVALCANTI al

LESÕES

CERVICAIS NÃO

CARIOSAS: UM

PANORAMA ATUAL

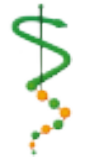

REV, ODONTOL,

UNIV. CID, SÃo

PAULO

2012; $24(1): 57$.

66 , JAN-ABR 
XAVIER AFC

PINTO TCA CAVALCANTI AL LESÕES CERVICAIS NÃO CARI OSAS: UM PANO-

RAMA ATUAL

52. Ferreira S, Sampaio J, Sampaio A. Sensibilidade dentinária: etiologia e diagnóstico diferencial Rev ABO Nac 2001 abr./maio;9(2):85-7.

53. Vale I, Bramante A. Hipersensibilidade dentinária: diagnóstico e tratamento. Rev Odontol Univ São Paulo 1997 jul./set.;11(3):207-13.

54. Conceição E. Dentística: saúde e estética. Porto Alegre: Artes Médicas; 2000.

Recebido em: 22/03/2012

Aceito em: 28/03/2012 